\title{
Timing of interfering events in one-trial serial overshadowing of a taste aversion
}

\author{
Dorothy W. S. Kwok ${ }^{1}$ - Justin A. Harris ${ }^{1}$ - Robert A. Boakes ${ }^{1}$
}

Published online: 23 August 2016

(C) Psychonomic Society, Inc. 2016

\begin{abstract}
This set of experiments examined the question of when a stimulus would be most effective in overshadowing the acquisition of long-delay taste aversion learning. In Experiment 1 rats drank sucrose, the target solution, followed by a hydrochloric acid $(\mathrm{HCl})$ solution before lithium injection some time later; $\mathrm{HCl}$ was presented either early or late in the interval. The late condition produced greater overshadowing than the early condition. The importance of the $\mathrm{HCl}$-injection interval was confirmed by Experiment 2, in which the sucrose-injection interval was varied. Experiment 3 found that even placement in a different context - an event that normally produces little overshadowing of a CTA - produced one-trial overshadowing of a sucrose aversion as long as the context was novel and exposure to it occurred immediately before lithium injection. No current theoretical account of one-trial overshadowing predicts that a late event produces more overshadowing than an early event. This result can, however, be accommodated within a modified version of the RescorlaWagner model.
\end{abstract}

Keywords Conditioned taste aversion - Long-delay learning - Serial overshadowing - Retroactive interference . Context conditioning

Serial overshadowing in one-trial taste aversion learning has been found in a number of studies. As Revusky (1971) first

Robert A. Boakes

bob.boakes@sydney.edu.au

1 School of Psychology (A18), University of Sydney, Sydney, NSW 2006, Australia demonstrated, giving a rat an additional taste during the interval between a target taste (conditioned stimulus or CS) and an injection of lithium chloride ( $\mathrm{LiCl}$ as the unconditioned stimulus or US) can strongly interfere with acquisition of an aversion to the target taste. According to some accounts of overshadowing, notably that proposed by Rescorla and Wagner (1972), at least two conditioning trials should be required for overshadowing to be detected. Yet many studies of one-trial conditioning have reported reliable overshadowing both in taste preparations (e.g., Kwok, Livesey, \& Boakes, 2012) and other conditioning paradigms (e.g., Mackintosh, 1971; Mackintosh \& Reese, 1979).

In addition to its theoretical interest, the study of overshadowing in taste aversion learning is important because of its potential application to the problems of anticipatory nausea and development of food and other aversions experienced by cancer patients undergoing chemotherapy. The introduction of a novel tasting food or drink just before administration of chemotherapy can reduce such side-effects by acting as a "scapegoat" that overshadows conditioning to aspects of the therapeutic setting (Quinn \& Colagiuri, 2015).

The central question addressed in the present study is: When does the introduction of a second taste within the interval between the target taste and $\mathrm{LiCl}$ injection produce the greatest one-trial overshadowing of an aversion to the target taste? Only two previous studies have addressed this question. As in the present study, these varied the timing of the interfering stimulus within the target taste-injection interval and assessed the extent to which an aversion to the target taste was overshadowed. In their sixth experiment Cannon, Best, Batson, Brown, Rubenstein, and Carrell (1985) presented a saccharin solution that was followed either immediately, 15 or $30 \mathrm{~min}$ later by 5 -min access to a coffee-flavored solution. All rats were then given a $\mathrm{LiCl}$ injection 35 min after the saccharin presentation. Overshadowing of the saccharin 
aversion was found to be greatest in the group that received the coffee closer to $\mathrm{LiCl}$. The authors noted the inverse relationship between the strength of the aversion to the target (saccharin) and the strength of the aversion to the interfering event (coffee), suggesting that this was consistent with theories based on competition between stimuli for associative strength. They also noted that the results were not consistent with the dominant theory of this type, in that the RescorlaWagner model predicts that overshadowing requires more than a single conditioning trial, as mentioned above and discussed further in the General Discussion below. However, Cannon et al. (1985) did not make any further suggestions as to how their results could be explained.

In the second study to examine timing of an interfering event in long-delay taste aversion learning, Kaye, Gambini, and Mackintosh (1988) gave rats vinegar as the target solution and then a sucrose solution either immediately or $3.5 \mathrm{~h}$ later; $\mathrm{LiCl}$ was injected $4 \mathrm{~h}$ post vinegar. Overshadowing by sucrose was found only when sucrose was given $3.5 \mathrm{~h}$ after the vinegar, a result consistent with that obtained by Cannon et al. (1985). Kaye et al. (1988) considered two possible explanations for this result. One was based on Wagner's (1981) SOP model. As also discussed further in the General Discussion, this proposes that in order for two or more stimuli to become associated their representations must be activated into a focal memory or primary state (A1) at the same time; subsequently they decay into an active, but secondary memory state (A2). Importantly, because there is a limit to A1, the later arrival of another stimulus and activation into A1 of its representation may accelerate decline of the target representation into its A2 state: "it is conceivable that ..... the delayed distractor might be more effective in displacing the representation of vinegar from the A1 state" (Kaye et al., 1988; p.45). And, if vinegar is no longer in an A1 state when lithium-induced sickness occurs, little or no aversion to vinegar will be acquired. However, they rejected this possibility because Wagner's (1981) theory could not also explain data on habituation obtained from the same set of experiments.

The second explanation considered by Kaye et al. (1988) was based on Pearce's (1987) configural theory. This proposes that an animal responds to a set of stimuli on a conditioning trial as if it had been presented with a single stimulus. Thus, Kaye et al. (1988) suggested that in their experiment both groups given vinegar and then sucrose (and $4 \mathrm{~h}$ later injected with $\mathrm{LiCl}$ ) formed a vinegar-sucrose configuration but the relative contribution of sucrose was greater in the group given sucrose after a 3.5-h delay. As a result, in the latter group there would be weaker generalization of the vinegar-sucrose aversion to vinegar in the subsequent test.

In a related study Kwok, Livesey, and Boakes (2012) gave rats saline either before (proactive interference) or after (retroactive interference) sucrose as the target taste. The results suggested that, at least for the parameters used in these experiments, two conditioning trials were needed to obtain proactive interference, whereas retroactive interference could be obtained after a single conditioning trial. To explain onetrial retroactive interference, these authors suggested that the associability of the target taste is reduced by the occurrence of a subsequent taste because the latter disrupts memory consolidation for the target taste. According to this account, the longer the target taste has been in memory, the more resistant it is to interference. This account therefore predicts that, as the interval between the target and distractor is extended, the interfering taste will have a weaker overshadowing effect.

In summary, it is not easy to explain results indicating that a late stimulus produces greater overshadowing than an early stimulus within a single pairing of a target taste with illness. Both the memory consolidation account, outlined above, and, as explained later, the influential SOP theory (Wagner, 1981) predict the opposite pattern of results. And for configural theory (e.g., Pearce, 1987) to explain these differences in serial overshadowing, it must be elaborated to allow configural processing of serially presented elements. It needs also to assume that the salience of serially occurring elements in a configural representation is a direct function of their recency at the time of reinforcement.

In view of the theoretical importance of the timing question, we decided to revisit this issue in preliminary experiments using our previous procedure whereby sucrose served as the target taste and saline as the interfering taste (Kwok et al., 2012). Following presentation of the target taste (sucrose) in drinking chambers located in a different room from their home cages, rats were returned to their home cages for at least $10 \mathrm{~min}$ before returning to the drinking chambers to be given access to the second, potentially overshadowing taste.

The preliminary experiments yielded conflicting results with respect to when an interfering taste produces greatest overshadowing. The possible reason for this inconsistency was suggested by two further unpublished experiments. These indicated that, when sucrose is used as the target stimulus and saline as the serial overshadowing stimulus in a longdelay CTA preparation, an aversion to sucrose can be inflated by generalization to sucrose from a saline aversion. Such generalization to sucrose was not found when a hydrochloric acid $(\mathrm{HCl})$ solution was used instead of saline. Consequently, in the first two experiments reported here the overshadowing stimulus was $\mathrm{HCl}$.

\section{Experiment 1}

Experiment 1 addressed the question of whether greater overshadowing by $\mathrm{HCl}$, the distractor taste, of an acquired aversion to sucrose, the target taste, would be produced by presenting HCL either shortly after sucrose or after a longer 
interval, during a single conditioning trial in which sucrose was followed by lithium injection $(\mathrm{LiCl}) 65$ min later. As shown in Table 1, for two of the groups, Early Distractor and Late Distractor, $\mathrm{HCl}$ was either presented $10 \mathrm{~min}$ or 50 min after sucrose, while a Control group received water during the 65 -min delay period. Half the animals in the Control group received water at the same time as the Early Distractor group received $\mathrm{HCl}$ and the other half at the same time as the Late Distractor group received $\mathrm{HCl}$.

A second aim of Experiment 1 was to discover whether one-trial overshadowing of a sucrose aversion might be found using a novel context. To date, only tastes have been found to produce one-trial overshadowing within a taste-illness paradigm. In a previous study overshadowing of a sucrose aversion was obtained when rats were placed in a novel context (steel cage) 10 min prior to being given sucrose in our standard drinking chambers; however, this effect appeared only after two conditioning trials (Kwok et al., 2012; Experiment 2). A subsequent unpublished experiment produced a similar outcome when rats were placed in the novel context $10 \mathrm{~min}$ after drinking the sucrose solution but again overshadowing was revealed only after two conditioning trials. However, if the closer an event is to $\mathrm{LiCl}$ injection, the stronger is the onetrial overshadowing effect it produces, this would suggest the possibility that one-trial overshadowing by a context might be detected if the context were experienced late in the sucrose$\mathrm{LiCl}$ interval. Therefore, as shown in Table 1, the fourth group in this experiment was a Context group, whereby these rats were placed in a novel context for 5 min immediately before injection. This timing was chosen with the aim of maximizing the possibility of obtaining overshadowing by a context.

\section{Method}

\section{Subjects}

Thirty-two male albino Wistars (Rattus norvegicus) were originally obtained from the University of Adelaide and had previously undergone instrumental training, in which none of the stimuli were tastes and no aversive treatment was administered. At the start of the present experiment they were approximately 100 days old and weighed a mean of $502 \mathrm{~g}$ (range 361-592 g). They were first adapted to a restricted drinking schedule whereby access to water in the colony room was progressively reduced from $4 \mathrm{~h}$ to $30 \mathrm{~min}$ over a 4 -day period. Once training commenced they received 30-min access to water immediately after returning to their home cages.

\section{Apparatus and solutions}

A separate laboratory contained sixteen transparent acrylic cages, $33 \times 21 \times 19 \mathrm{~cm}$ high, referred to here as drinking chambers. Flooring was commercial cat litter. Plastic drinking bottles of $100-\mathrm{ml}$ capacity with a stainless steel ball-bearing spout could be attached to the drinking chambers using a metal bracket, entering at a $45^{\circ}$ angle though the steel wire roof. The novel context consisted of a set of sixteen steel cages with steel rod flooring, $20 \times 20 \times 30 \mathrm{~cm}$ high. Intakes were measured by weighing bottles to the nearest $0.1 \mathrm{~g}$ before and after each session. The target solution was $8 \%$ sucrose (CSR brand; www.csrsugar.com.au) and the interfering solution was a $0.005 \mathrm{M} \mathrm{HCl}$ solution, both mixed with tap water.

Table 1 Summary of designs of Experiments 1-3. The Control group in each experiment was subdivided so that the timing of events for each subgroup corresponded to that in an experimental group. No differences in water consumption were found between these sub-groups

\begin{tabular}{|c|c|c|c|c|c|}
\hline & & Pre-exposure & Conditioning & Test & \\
\hline \multicolumn{6}{|c|}{ Experiment 1} \\
\hline \multirow[t]{3}{*}{ Groups } & $\begin{array}{l}\text { Early Distractor } \\
\text { Late Distractor }\end{array}$ & $\mathrm{n} / \mathrm{a}$ & $\begin{array}{l}\text { Sucrose } \rightarrow(10) \rightarrow \mathrm{HCI} \rightarrow(50) \rightarrow \mathrm{LiCI} \\
\text { Sucrose } \rightarrow(50) \rightarrow \mathrm{HCI} \rightarrow(10) \rightarrow \mathrm{LiCI}\end{array}$ & $1 \times$ Sucrose & $1 \times \mathrm{HCI}$ \\
\hline & Context & & Sucrose $\rightarrow(60) \rightarrow$ Context $\rightarrow(0) \rightarrow$ LiCI & & \\
\hline & Control & & Sucrose $\rightarrow$ Water $\rightarrow$ LiCI & & \\
\hline \multicolumn{6}{|c|}{ Experiment 2} \\
\hline \multirow[t]{2}{*}{ Groups } & $\begin{array}{l}65 \\
95\end{array}$ & $\mathrm{n} / \mathrm{a}$ & $\begin{array}{l}\text { Sucrose } \rightarrow(50) \rightarrow \mathrm{HCI} \rightarrow(10) \rightarrow \mathrm{LiCI} \\
\text { Sucrose } \rightarrow(50) \rightarrow \mathrm{HCI} \rightarrow(40) \rightarrow \mathrm{LiCI}\end{array}$ & $1 \times$ Sucrose & $1 \times \mathrm{HCI}$ \\
\hline & Control & & Sucrose $\rightarrow$ Water $\rightarrow$ LiCI & & \\
\hline \multicolumn{6}{|c|}{ Experiment 3} \\
\hline \multirow[t]{3}{*}{ Groups } & $\begin{array}{l}\text { Late Distractor } \\
\text { Contiguous Distractor }\end{array}$ & $\begin{array}{l}\text { A A A } \\
\text { A A A }\end{array}$ & $\begin{array}{l}\text { Sucrose } \rightarrow(50) \rightarrow \text { Place in } \mathrm{B} \rightarrow(10) \rightarrow \text { LiCI } \\
\text { Sucrose } \rightarrow(60) \rightarrow \text { Place in } \mathrm{B} \rightarrow(0) \rightarrow \text { LiCI }\end{array}$ & $2 \times$ Sucrose & \\
\hline & Pre-exposed Distractor & B B B & Sucrose $\rightarrow(60) \rightarrow$ Place in $\mathrm{B} \rightarrow(0) \rightarrow$ LiCI & & \\
\hline & Control & A A A & Sucrose $\rightarrow$ Place in $\mathrm{A} \rightarrow \mathrm{LiCI}$ & & \\
\hline
\end{tabular}

Note: Numbers in parenthesis indicate time spent in home cages in between access to fluids in drinking chambers

$\mathrm{HCl}$ hydrochloric acid solution, $\mathrm{LiCl}$ lithium injection 


\section{Procedure}

Experimental sessions were conducted 7 days per week. During water pre-training on Days 1-3 all rats were given water in the drinking chambers, with access progressively reduced from 20 to $10 \mathrm{~min}$. From then on all sessions lasted $10 \mathrm{~min}$, unless otherwise specified below. Rats were matched for water intake on Day 3 when allocated to the four groups (each $n=8$ ).

In the single conditioning session (Day 4) all rats were first given sucrose for $5 \mathrm{~min}$ in the drinking chambers, returned to their home cages, and $65 \mathrm{~min}$ later were given an injection of $0.15 \mathrm{M} \mathrm{LiCl}$ at $10 \mathrm{ml} / \mathrm{kg}$. The groups differed only as to events during this delay period. The Early Distractor group was given 5-min access to $\mathrm{HCl}$ in the drinking chambers 10 min postsucrose and then returned to the home cages, whereas the Late Distractor group received the same treatment 50 min postsucrose. The Control group was given water instead of $\mathrm{HCl}$ : Half of these rats were given water 10 min post-sucrose, while the other half were given water $50 \mathrm{~min}$ post-sucrose. The Context rats were placed in the steel chambers for $5 \mathrm{~min}$ 60 min post-sucrose and on removal were injected with minimal delay. All sucrose, water and $\mathrm{HCl}$ amounts were limited to approximately $6 \mathrm{ml}$.

On Days 5 and 6 all rats received 10-min access to water in the drinking chambers exactly as during pre-training. On Day 7 rats were given access to sucrose for $10 \mathrm{~min}$. Rats were given a similar session on Day 8 with access to the $\mathrm{HCl}$ solution.

\section{Results}

Water intakes on the final pre-training day (Day 3) did not differ between groups when the mean (and SEM) intakes were: Early Distractor, 11.6 (0.9) $\mathrm{ml}$; Late Distractor, 11.9 (1.1) $\mathrm{ml}$; Context, 12.4 (1.0) $\mathrm{ml}$; and Control, 10.6 (0.6) $\mathrm{ml}$. This was confirmed by an ANOVA, which detected no effect of Group, $F<1$. Day 6 water intakes were also analyzed for differences, which failed to reveal any Group effect, $F<1$. Both these intakes - in the session immediately prior to conditioning and the session prior to the first test session - were compared to check that no aversions to the drinking chambers were found across sessions and groups. A $4 \times 2$ mixed ANOVA did not detect any main effects of group or Session, or any interactions, largest $F$ $<1$. Thus, water intakes effectively did not change from before to after the conditioning treatment and there were no differences between the groups on this measure. These results suggest that the rats had not acquired any appreciable aversion to the drinking chambers. Furthermore, no difference in water consumption during conditioning was found between the Control subgroups, $F<1$. Therefore, these data have been combined for a single Control group.

Sucrose intakes in the conditioning session (Day 4) and in the sucrose test session (Day 7) are shown in Fig. 1a. Whereas no group differences in sucrose consumption were found on Day $4, F(3,28)=1.11, p=.36$, the same one-way ANOVA applied to sucrose test intakes (Day 7) did reveal group differences, $F(3,28)=13.42, p<.001$, $\eta_{\mathrm{p}}{ }^{2}=0.59\left(95 \%\right.$ confidence interval on $\left.\eta_{\mathrm{p}}{ }^{2}=0.28-0.71\right)$. An a priori contrast confirmed that sucrose intakes in the Early and Late Distractor groups were greater than those in the Control group, $F(1,28)=33.72, p<.001, \eta_{\mathrm{p}}{ }^{2}=$ $0.55(0.27-0.69)$, thus indicating one-trial overshadowing by $\mathrm{HCl}$ of the sucrose aversion. Of major interest was the finding that Late Distractor rats drank significantly more sucrose than did Early Distractor rats, $F(1,28)=4.64, p=$ $.04, \eta_{\mathrm{p}}{ }^{2}=0.14(0.00-0.37)$. The final important finding from the sucrose test was that Context rats drank more than Controls, $F(1,28)=4.95, p=.034, \eta_{\mathrm{p}}{ }^{2}=0.15$ (0.00-0.38).

Intakes of $\mathrm{HCl}$ are shown Fig. 1b. For the Early and Late Distractor groups, the strength of a group's $\mathrm{HCl}$ aversion was the inverse of its aversion to sucrose. Thus, $\mathrm{HCl}$ intakes were smaller in the Late Distractor group than in the Early Distractor group, while Early Distractor intakes were nonetheless smaller than Control intakes. A priori contrasts confirmed this description, with the two Interval groups combined consuming less $\mathrm{HCl}$ than Controls and Context rats, $F(1,28)=$ $9.26, p=.005, \eta_{\mathrm{p}}{ }^{2}=0.25(0.03-0.47)$. No difference between Context and Control groups was found, $F<1$, as expected, since $\mathrm{HCl}$ was novel at test for both these groups. The Interval groups each consumed less $\mathrm{HCl}$ than did Controls, $F(1,28)=$ 4.42, $p=.036, \eta_{\mathrm{p}}{ }^{2}=0.14(0.00-0.36)$, and $F(1,28)=7.36, p=$ $.011, \eta_{\mathrm{p}}{ }^{2}=0.21(0.01-0.43)$, respectively. Finally, the Early Distractor group drank more $\mathrm{HCl}$ than did the Late Distractor group, $F(1,28)=4.32, p=.029, \eta_{\mathrm{p}}{ }^{2}=0.13(0.00-0.36)$.

\section{Discussion}

The most important result from this experiment was that overshadowing was greater when the $\mathrm{HCl}$ was presented late in the interval, i.e. with only a 10-min delay before $\mathrm{LiCl}$ injection, than when presented early, i.e. only $10 \mathrm{~min}$ after the sucrose. The second important result was that the novel context also produced one-trial overshadowing. In relation to our previous attempts to obtain such an effect when the context was experienced earlier in the delay, this supports the idea that stimuli that are normally ineffective can overshadow acquisition of a taste aversion if they occur sufficiently late in the taste- $\mathrm{LiCl}$ delay interval. This suggestion was later tested in Experiment 3. 
A

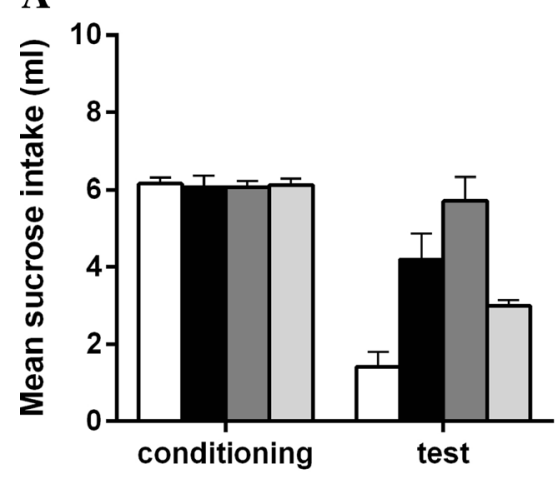

Fig. 1 Experiment 1. (a) Sucrose intakes during the single conditioning session and test session. Lithium was injected 65 min after sucrose. $\mathrm{HCl}$ was given either $10 \mathrm{~min}$ after sucrose in the Early Distractor group, 50 min after sucrose in the Late Distractor group or given water instead

\section{Experiment 2}

The results from Experiment 1 left open the question of whether the interval between the $\mathrm{HCl}$ and $\mathrm{LiCl}$ injection was the important factor in determining the degree of overshadowing, as opposed to the interval between sucrose and $\mathrm{HCl}$. Consequently, in Experiment 2 a group for which access to sucrose was followed by an early $\mathrm{LiCl}$ injection (65 min) was compared with a group given a late injection (95 min); for both groups the interval between sucrose and $\mathrm{HCl}$ was held constant at $50 \mathrm{~min}$. A control group was included that was given only water during the sucrose- $\mathrm{LiCl}$ interval. The experimental design is shown in Table 1.

\section{Method}

\section{Subjects}

Twenty-four experimentally naïve male Albino Wistar rats were obtained from the Animal Resources Centre (ARC) Perth, Western Australia. They were 70 days old at the start of the experiment, when their mean weights were $325.9 \mathrm{~g}$ (range 267-375 g). Housing, food, water restriction and other general procedures were the same as the previous experiments.

\section{Apparatus and solutions}

The drinking chambers and solutions were those used in Experiment 1.

\section{Procedure}

Rats received water pre-training on Days $1-3$, as previously described. From then on all sessions lasted $10 \mathrm{~min}$, unless

\section{B}

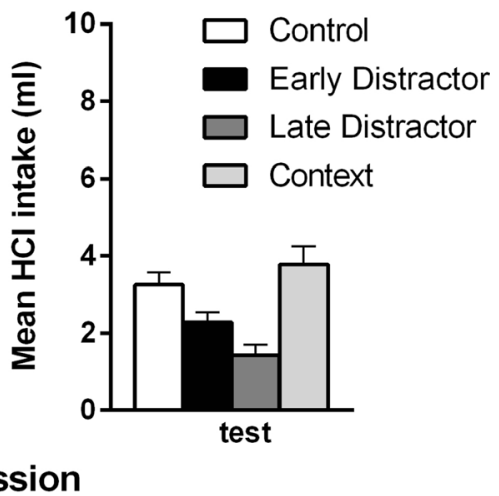

(Control group). Rats in the Context group were placed in a novel context for 5 min immediately prior to injection. (b) Hydrochloric acid intakes in the single $\mathrm{HCl}$ test session. Means and SEMs are shown

otherwise specified below. Rats were matched for water intake on Day 3 when allocated to the three groups (each $n=8$ ).

The groups were labeled 65, 95, and Control. In the single conditioning session (Day 4) rats in the experimental groups (65 and 95) were placed in the drinking chambers where they had access to sucrose for $5 \mathrm{~min}$, returned to their home-cages in the colony room for $50 \mathrm{~min}$ and then given $\mathrm{HCl}$ for $5 \mathrm{~min}$ in the drinking chambers. The 65 group was given a $0.15 \mathrm{MLiCl}$ injection at $10 \mathrm{ml} / \mathrm{kg}$ of a rat's bodyweight $65 \mathrm{~min}$ after sucrose, while the 95 group was given the same injection $95 \mathrm{~min}$ after sucrose. Rats in the Control group were placed into the drinking chambers to consume sucrose for $5 \mathrm{~min}$ and returned to their home cages. These rats received access to $5 \mathrm{~min}$ of water $50 \mathrm{~min}$ post-sucrose and then half were injected $65 \mathrm{~min}$ post-sucrose, while the other half were injected 95 min post sucrose. All solutions during conditioning were limited to approximately $6 \mathrm{ml}$.

On Days 5 and 6 rats were given water in the drinking cages. A single sucrose test was given on Day 7 , when rats were given access to sucrose for $10 \mathrm{~min}$. This was followed by 10-min access to $\mathrm{HCl}$ on Day 8.

\section{Results}

The groups did not differ in terms of water intakes either before conditioning or before the sucrose test (Days 3 and 6). Mean (and SEM) water intakes on Day 3 were: Group 65, 12.9 (0.6) ml; Group 95, $12.7(0.8) \mathrm{ml}$; and Control, $13.8(0.9) \mathrm{ml}$. ANOVA applied to these data did not detect any Group effects on any of these water days, largest $F<1$. A further $3 \times 2$ mixed ANOVA found no differences across these Sessions, between Groups, nor any interaction, $F<1$.

Preliminary analyses failed to detect any differences between the two control sub-groups and so their data were combined in the analyses reported next. Sucrose intakes in the 
conditioning session (Day 4) and in the sucrose test session (Day 7) are shown in Fig. 2a. No group differences in sucrose consumption were found on Day $4, F(2,21)=3.07, p>.05$, but the same one-way ANOVA applied to sucrose test intakes (Day 7) did reveal group differences, $F(2,21)=11.99, p<$ $.001, \eta_{\mathrm{p}}{ }^{2}=0.53\left(95 \%\right.$ confidence interval on $\eta_{\mathrm{p}}{ }^{2}=0.17$ 0.69). Planned orthogonal contrasts confirmed that sucrose intakes in the groups that received $\mathrm{HCl}$ during the delay (65 and 95) were greater than those in the Control group, $F(1,21)$ $=19.27, p<.001, \eta_{\mathrm{p}}{ }^{2}=0.48(0.15-0.66)$, thus indicating onetrial overshadowing by $\mathrm{HCl}$ of the sucrose aversion. Importantly, Group 65 drank significantly more sucrose than did Group 95, $F(1,21)=4.70, p=.042, \eta_{\mathrm{p}}{ }^{2}=0.18(0.00$ 0.44).

Intakes of $\mathrm{HCl}$ are shown in Fig. 2b. A one-way ANOVA applied to $\mathrm{HCl}$ intakes revealed significant group differences, $F(2,21)=25.27, p<.001, \eta_{\mathrm{p}}{ }^{2}=0.98(0.00-0.98)$. A priori contrasts confirmed this description, with the two overshadowing groups (65 and 95) consuming less $\mathrm{HCl}$ on average than Controls, $F(1,21)=45.86, p<.001, \eta_{\mathrm{p}}{ }^{2}=0.69$ $(0.40-0.80)$. As in Experiment 1, the strength of a group's aversion to $\mathrm{HCl}$ was the inverse of its aversion to sucrose, such that $\mathrm{HCl}$ intakes were smaller in Group 65 than in Group 95, $F(1,21)=5.28, p=.032, \eta_{\mathrm{p}}{ }^{2}=0.20(0.00-0.45)$.

\section{Discussion}

The main result from this experiment was to establish the importance of the $\mathrm{HCl}$-injection interval, in that overshadowing of the sucrose aversion was greater in Group 65, which experienced a short $\mathrm{HCl}$-injection interval, than in Group 95, which had a long HCl-injection interval. The finding in both Experiment 1 and the present experiment that the strengths of the sucrose and $\mathrm{HCl}$ aversions were complementary suggests that the degree to which a stimulus overshadows the target stimulus depends on how strongly the overshadowing stimulus is associated with the same outcome. This suggestion is expanded in the General Discussion below.

\section{Experiment 3}

Experiment 1 detected an overshadowing effect when during the conditioning session rats were placed in a different context to that in which they had previously received both water and sucrose. In discussing this result we suggested that this probably depended on the overshadowing context being both novel and experienced immediately prior to injection. The aim of Experiment 3 was to test this suggestion. Given that a context is generally less associable with nausea than a taste (e.g., Kwok et al., 2012), we suspected that even the introduction of an interval as short as 10 min between a novel context and lithium injection might not produce overshadowing, even though overshadowing by a taste was found in Experiment 2 with an interval as long as $40 \mathrm{~min}$. To examine this possibility, rats were placed in a novel context either immediately before lithium injection (under the exact parameters for the Context group in Experiment 1) or 10 min before injection. These two groups were labelled Contiguous Distractor and Late Distractor, respectively. A third group, Pre-exposed Distractor, was placed in the different context prior to conditioning in order to test whether overshadowing by this context depends on whether in the conditioning session it is novel or not. Finally, a Control group was included that did not experience any relevant potentially overshadowing event in the conditioning session. The design of Experiment 3 is summarized in Table 1.
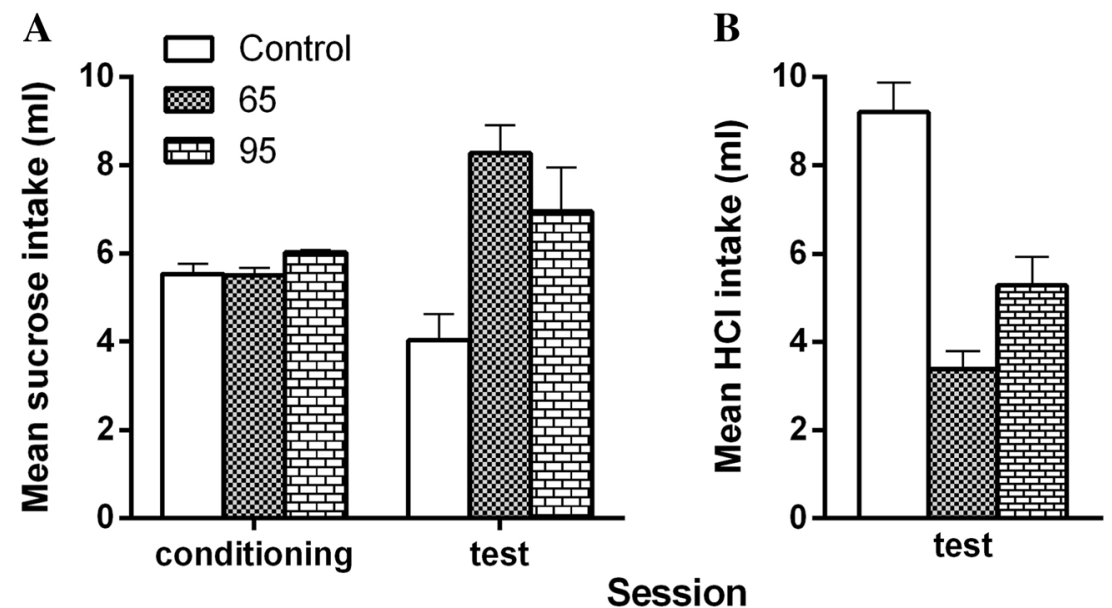

Fig. 2 Experiment 2. (a) Sucrose intakes during the single conditioning session and test session. Lithium was injected 65 or 95 min post sucrose. $\mathrm{HCl}$ was given $50 \mathrm{~min}$ after sucrose for both $\mathrm{HCl}$ groups, and at the same

\section{Session}

time, water was given to the Control group. (b) Hydrochloric acid intakes in the single $\mathrm{HCl}$ test session. Means and SEMs are shown 


\section{Method}

\section{Subjects}

Forty experimentally naïve male Albino Wistar rats were obtained from ARC Perth. At the start of the present experiment they were 70 days old and weighed a mean of $341 \mathrm{~g}$ (range 311-369 g). Housing, food, water restriction, and other general procedures were the same as in Experiment 1.

\section{Apparatus and solutions}

The drinking chambers (acrylic cages) and the novel context (steel cages) were those used in Experiment 1. The sucrose solution also remained unchanged at $8 \%$.

\section{Procedure}

Rats received water pre-training on Days $1-3$ and were matched for water intakes on Day 3 when allocated to the four groups (each $n=10$ ). All sessions after pre-training lasted $10 \mathrm{~min}$, unless otherwise specified below.

In the three pre-exposure sessions (Days 4-6) the Preexposed Distractor group were placed in the steel cages for $5 \mathrm{~min}$ to consume water, while the remaining groups were placed in the regular drinking chambers to consume water for the same amount of time. Thus, the steel cages would not be novel for the Pre-exposed Distractor group in the subsequent conditioning session.

In the single conditioning session (Day 7) all rats were first given sucrose for $5 \mathrm{~min}$ in the drinking chambers, returned to their home cages and 65 min later were given an injection of $0.15 \mathrm{M} \mathrm{LiCl}$ at $10 \mathrm{ml} / \mathrm{kg}$. Sucrose consumption was limited to $6 \mathrm{ml}$ during this session. The groups differed only as to events during this delay period. Late Distractor rats were placed for 5 -min in the steel cages 50 min post-sucrose and then returned to their home cages, whereas Contiguous Distractor rats were placed in the steel cages 60 min post-sucrose and were injected as soon as they were removed from this context, thus replicating conditions for the Context group in Experiment 1. The Pre-exposed Distractor group was also placed in the steel cages $60 \mathrm{~min}$ post-sucrose and injected on removal. Finally, half of the Control rats were placed in the drinking chambers 50 min post-sucrose, while the remaining rats were placed in the drinking chambers $60 \mathrm{~min}$ post-sucrose.

On Days 8 and 9 all rats received 10 min of water in the drinking chambers. On Day 10 and Day 16 rats were given unlimited access to sucrose for $10 \mathrm{~min}$. During the 5-day interval between these test sessions (Days 11-15) rats remained in their home-cages where they were given 30-min water daily. This second test allowed examination of whether any overshadowing effect would persist over a delay period.

\section{Results}

Mean (and SEM) water intakes for the four groups on Day 3 were: Late Distractor, 10.7 (0.7) ml; Contiguous Distractor, 10.8 (0.5) ml; Pre-exposed Distractor, 12.2 (0.6) $\mathrm{ml}$; and Control, $11.0(0.8) \mathrm{ml}$. No group differences in water intakes were detected either in the final pre-training session (Day 3) or in the session before the sucrose test (Day 9), largest $F=1.06$, $p>.05$. Furthermore, although a $4 \times 2$ mixed ANOVA examining intakes between these two sessions detected a main effect of Session, $F(1,36)=16.66, p<.001, \eta_{\mathrm{p}}{ }^{2}=0.32(95 \%$ confidence interval on $\left.\eta_{\mathrm{p}}{ }^{2}=0.09-0.51\right)$, no main effect of Group or Session by Group interaction were found, both $F \mathrm{~s}$ $<1$. Thus, no evidence for a context aversion to the drinking chambers or any group differences in water intakes were detected.

Sucrose intakes in the conditioning session (Day 7) and sucrose test sessions (Day 10 and Day 16) are shown in Fig. 3. Unsurprisingly, given that intakes were capped, no group differences were found on Day 7 by a one-way ANOVA, $F<1$. A mixed ANOVA examining intakes over the two test sessions revealed main effects of Session and Group, $F(1,36)=74.06, p<.001, \eta_{\mathrm{p}}{ }^{2}=0.67(0.47-0.77)$, and $F(3,36)=6.51, p=.001, \eta_{\mathrm{p}}{ }^{2}=0.35(0.08-0.51)$, respectively. However, no Session by group interaction was found, $F$ $<1$, and consequently planned contrasts were applied to mean intakes over the two test sessions. An a priori contrast between rats placed in novel steel cages (Context B) in the conditioning session (the Contiguous rats) and Control groups confirmed that sucrose consumption was significantly greater in the Contiguous group, $F(1,36)=15.39, p<.001, \eta_{\mathrm{p}}{ }^{2}=0.30$ (0.08-0.49), thus showing overshadowing of the sucrose aversion by this novel context. Similar contrasts between the Late

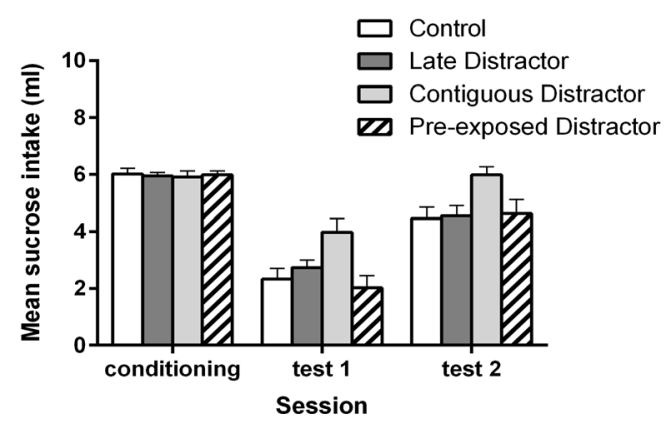

Fig. 3 Experiment 3. Sucrose intakes during the single conditioning session and test sessions. Lithium was injected 65 post sucrose and placement in a second context (steel cages) served as the interfering stimulus. Placement in this context was given either $50 \mathrm{~min}$ after sucrose (Late Distractor), immediately before injection (Contiguous and Pre-exposed Distractor) or not at all (Control); instead this last group was placed in the familiar drinking chambers. The Pre-exposed Distractor group had been placed in the steel cages on three occasions prior to the conditioning session, whereas for the Late and Contiguous Distractor groups this context was first experienced in the conditioning session. Means and SEMs are shown 
Distractor and Control group, and the Pre-exposed Distractor and Controls, failed to find any differences between either of these comparisons, $p>.015$, using the Bonferroni adjustment. Thus, only the Contiguous Distractor rats differed from the Controls. Importantly, rats that had been pre-exposed to the steel cages (Pre-exposed Distractor group) drank less sucrose than those who had experienced this context for the first time during conditioning (Late and Contiguous Distractor groups), $F(1,36)=7.05, p=.012, \eta_{\mathrm{p}}{ }^{2}=0.16(0.01-0.37)$. The final important finding was that Contiguous Distractor rats drank more sucrose on test than Late Distractor rats, $F(1,36)=8.64$, $p=.006, \eta_{\mathrm{p}}^{2}=0.19(0.02-0.40)$. The pattern of significant results was identical in an analysis using the full set of orthogonal contrasts. It may be noted that these differences are not always obvious in Fig. 3, since this shows data from each test session, whereas the planned contrasts were applied to intakes averaged over both session.

\section{Discussion}

This experiment provides direct evidence supporting the suggestion from Experiment 1 that for a context to produce a onetrial overshadowing effect it needs to be both novel and be experienced immediately before injection. The present results suggest a potential methodological problem for conditioned taste experiments in which rats are transferred to a novel context to be injected. Such a procedure may result in a weaker taste aversion than if the injections took place in a familiar setting.

\section{General discussion}

The general question addressed by this set of experiments was: What timing of an interfering stimulus produces greatest serial overshadowing of conditioning to the target stimulus? The results from Experiments 1 and 3 provide the answer: The later that a stimulus occurs within a target-US interval, the greater the overshadowing it produces. Thus, in Experiment $1 \mathrm{HCl}$ produced greater overshadowing when experienced only $10 \mathrm{~min}$ before the $\mathrm{LiCl}$ injection than when presented 50 min before injection and in Experiment 3 a novel context produced greater overshadowing when experienced immediately before injection than when experienced $10 \mathrm{~min}$ before injection. Furthermore, Experiment 2 confirmed that the interval between the interfering stimulus and lithium injection is the important timing factor. These results clearly rule out the consolidation theory proposed by Kwok et al. (2012). Whether or not they are consistent with the other theories outlined in the Introduction is the main concern of the discussion that follows.
The conclusion that late stimuli produce greater overshadowing is consistent with the two previous studies outlined in the Introduction. One advantage of the present Experiments 1 and 2 is that, by separating exposures to the target and overshadowing tastes with a period spent in the home cages, they reduced the likelihood of configuring the two tastes, compared to the Immediate conditions, whereby the target was followed immediately by the interfering taste, in Cannon et al. (1985) and by Kaye et al. (1988). Nonetheless, the observation that a late distractor produces more interference than an early one can be explained by a configural model of conditioning (e.g., Pearce, 1987), as argued by Kaye et al. (1988). The key feature of Pearce's model is that, on a conditioning trial, the associative strength of only one representation (configural unit) is updated. As Kaye et al suggested, if one assumes that the salience of the interfering stimulus depends on its recency at the time of reinforcement, then a late interfering stimulus will be more strongly represented within the configural unit than will an early interfering stimulus. As a result, the target stimulus will form only a small part of the configural unit when the interfering stimulus is late. At one extreme, the configural unit that undergoes conditioning may only represent the interfering stimulus, in which case conditioning to the target will fail completely.

While an extension of Pearce's (1987) configural model can explain why a target suffers more overshadowing from a late interfering stimulus than from an early one, it is less obvious that it can explain a key finding from Experiment 2. In that experiment overshadowing of sucrose was less (i.e., conditioning was greater) when the interval between the interfering stimulus and lithium was long than when it was short (40 min vs. $10 \mathrm{~min}$ ), even though the interval between the target and interfering stimuli was fixed $(50 \mathrm{~min})$. Therefore, in configural terms, delaying injection of lithium must have reinstated the representation of the target in the configural unit. This seems paradoxical given that delaying the lithium injection would further reduce the recency of the target. However, in Pearce's model, it is the relative salience of elements, rather than their absolute salience, that is important for their standing in the configural unit. As such, while the delay of lithium would presumably reduce the salience of the target stimulus, it would also reduce the salience of the interfering stimulus. If one assumes that the salience of these stimuli undergoes an exponential decay rate, then in absolute terms, the interfering stimulus would have lost more salience (because it had a higher initial value) than the target stimulus. Because of this absolute difference in loss of salience between the two tastes, the relative salience of the target taste would increase after the delay, and so its representation within the configural unit would also improve.

The other model that provides an explanation one-trial overshadowing is Wagner's (1981) influential SOP model. However, it is not easy to decide whether this model predicts 
when a stimulus will produce greater overshadowing under conditions like those of the present experiments. Consequently we carried out simulations of the model. In SOP conditioning depends on the simultaneous processing of CS elements and US elements in a primary activation state ("A1"). For trace conditioning to occur, there must be residual activity of CS elements in A1 when the US is presented, and this depends on the rate at which elements decay from A1 to a secondary activation state ("A2"). In SOP, elements decay from A1 due to capacity limits on that state, and the decay rate for elements of one stimulus is accelerated if another stimulus is presented that competes for A1 activity. This allows SOP to explain one-trial serial overshadowing, because the interfering stimulus will displace elements of the target stimulus from A1, thereby reducing the number of target elements in A1 at the time of the US.

Figure 4 shows simulations of A1 activation (based on Wagner, 1981) for elements of a target stimulus, presented for 10 time units, across a trace interval of 100 time units. In this simulation, the probability of self-generated activation of $\mathrm{CS}$ and distractor elements into A1 was set at 0.5 , the probabilities of decay from A1 and A2 were set at .001 and .0002, respectively, and the capacity parameter, $\mathrm{C} 1$, was 80 . These simulations show how the decay of target elements is accelerated by presentation of another (interfering) stimulus for 10 time units. Of particular relevance to the present discussion, the net impact of an interfering stimulus is greater if that stimulus is presented early rather than late in a trace interval. This

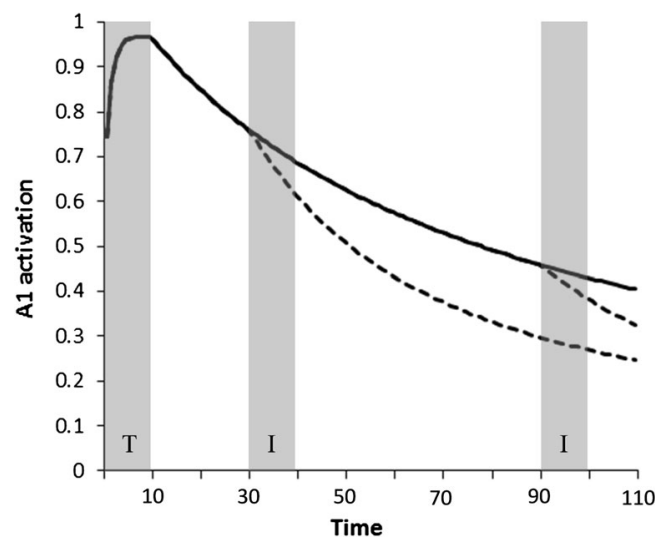

Fig. 4 Simulation of the A1 activation strength of a stimulus trace according to Wagner's (1981) SOP model. The plot shows the proportion of stimulus elements active in the A1 state as time elapses during and following presentation of a target $(\mathrm{T})$ for 10 time units. Upon termination of the target, T's elements begin to decay from the A1 state, as determined by the capacity limit of A1. The solid line shows this decay process across a 100 time unit trace interval when no other stimulus is presented. The dashed lines show how the decay of $\mathrm{T}$ elements is accelerated when an interfering stimulus (I), of the same size as $\mathrm{T}$, is presented for 10 time units, either early in the trace interval (20 time units after offset of $\mathrm{T}$ ) or late in the trace interval ( 80 time units after offset of T). The net activation of $\mathrm{T}$ elements at the end of the trace interval is lower when I occurs early than when it occurs late because the decay of $\mathrm{T}$ is accelerated over a longer time period in the former case is because, when the interfering stimulus occurs early, the target stimulus is subject to the accelerated decay rate over a longer portion of the trace interval. Therefore, when the interfering stimulus occurs early, there will be fewer target elements active in $\mathrm{A} 1$ at the end of the trace interval, and thus weaker conditioning of the target. Thus, the prediction from SOP, like that of Kwok et al. (2012), is opposite to the empirical evidence. Another problem for SOP is to explain the key result in Experiment 2, i.e. that delaying the injection of lithium served to restore conditioning of the target taste. This finding is difficult for SOP because the delay would increase the interval between the target and US, which could only serve to reduce A1 activation of the target at the time of reinforcement. If one were to imagine extending the dashed lines in Fig. 4, their path would continue to decline, and thus conditioning of the target should be weaker rather than stronger.

A notable feature of the results from Experiments 1 and 2 was that the relative strengths of the sucrose and $\mathrm{HCl}$ aversions were complementary. For example, the 95 condition of Experiment 2, where $\mathrm{HCl}$ was given $50 \mathrm{~min}$ after sucrose and the injection was given 95 min after sucrose, resulted in a moderate sucrose aversion but a weak $\mathrm{HCl}$ aversion, whereas the 65 condition, where $\mathrm{HCl}$ was again given 50 min after sucrose and the injection only $10 \mathrm{~min}$ after $\mathrm{HCl}$ was removed, resulted in a weak sucrose aversion but a strong $\mathrm{HCl}$ aversion. Such complementarity is consistent with two very different types of explanation for overshadowing. One approach, as formalized theoretically by Rescorla and Wagner in 1972, and discussed next, is to assume that decreased responding on test to the overshadowed stimulus reflects a failure of acquisition. We then discuss the second approach, one that assumes overshadowing to represent a performance deficit at test (e.g., Denniston, Savastano, \& Miller, 2001).

As noted at the beginning of this paper, the problem with applying the Rescorla-Wagner theory to the present results is that it does not predict overshadowing following a single conditioning trial. Thus, when the occurrence of lithium-induced nausea is entirely unpredicted, this leads to the formation of associations between preceding events and nausea such that, unlike in Wagner's SOP model, each component of a compound stimulus - e.g., sucrose followed by $\mathrm{HCl}$ in Experiments 1 and 2 - will gain as much associative strength as if it were conditioned in isolation. On the second trial the occurrence of nausea is more strongly predicted if in the first trial two or more stimuli had become associated with this outcome than if only one had; as a result, the increment in associative strength to the target-nausea association (and thus the acquired sucrose aversion) is smaller - because the error term is smaller - than if the target had occurred on its own.

One way of modifying the Rescorla-Wagner theory to allow for one-trial overshadowing is to assume that what the researcher regards as a single trial might for the animal functions as a series of mini-trials. Our suggested modification 
starts by assuming that both tastes and nausea are longerlasting events than events such as the tones or shocks used in other forms of conditioning. Thus, in the conditioning session the animal may over a minute or so intermittently recall the taste while continuing to experience nausea. Thus, to take as an example the 65 condition of Experiment 2, an initial pairing of $\mathrm{HCl}$ and nausea would produce a strong aversion to $\mathrm{HCl}$ that could serve to partially block acquisition of an association between subsequent recall of the sucrose memory and nausea some seconds later. This modification would then account for the observation that - even after a single conditioning session - the stronger the aversion to $\mathrm{HCl}$, the weaker the aversion to sucrose. To account for one-trial overshadowing by a novel context, as demonstrated in Experiments 1 and 3, one would need also to assume that memory for some features of the context - possibly its odor - are also more long-lasting than, say, a tone.

This account suggests that the present results may be restricted to taste aversion learning. An important test would be to use a one-trial trace fear conditioning procedure to determine the timing of a brief stimulus during the trace interval that would produce the greatest overshadowing effect and to determine whether this depended on use of a single or multiple shocks as the US. More generally, we note that there are parallels between the present results and some studies of overshadowing in a spatial domain. These have found that, in a task requiring either pigeons or humans to locate a hidden target in an array of potential targets, a cue ('landmark') close to the target can overshadow a more distant cue (e.g., Leising, Garlick, \& Blaisdell, 2011; Spetch, 1995.) However, such experiments have involved a very large number of training trials in contrast with the single conditioning session used in the present experiments, an advantage conveyed by the remarkable ability of rats and other species to acquire so rapidly an association between a taste and the delayed occurrence of some kind of malaise.

The second type of explanation of overshadowing is one assuming that, even with multiple trials, the acquisition of an association between a target stimulus and some outcome is unaffected by the presence of other stimuli. The latter are important only when the target stimulus is tested. At this point the response to the target stimulus is reduced to the extent that associated stimuli predict the same outcome (e.g., Denniston et al., 2001). This explanation of overshadowing provided by comparator theory is based on overshadowing procedures involving simultaneous compounds, so that the comparison that takes place at test depends on the formation during conditioning of within-compound associations between the target and overshadowing stimuli. Then, at test the target evokes the outcome both directly and indirectly via associative links with the overshadowing stimulus; the stronger the latter evokes the outcome, the weaker the conditioned response to the target stimulus.
This kind of "failure-of-performance" explanation does not work well when serial compounds are involved, as in the present experiments. In particular, when $\mathrm{HCl}$ is presented late in a sucrose-lithium interval, the within-compound association between sucrose and $\mathrm{HCl}$ would be much weaker than when the $\mathrm{HCl}$ is presented soon after the sucrose; indeed, attempts to detect an association between tastes separated by more than $10 \mathrm{~s}$ have been unsuccessful (Lavin, 1976). Thus, the stronger $\mathrm{HCl}$ aversion produced by its late presentation would at best be only weakly evoked on test by a sucrose$\mathrm{HCl}$ within-compound association. Perhaps more to the point, following Revusky, Parker, and Coombes (1977), Schachtman, Kasprow, Meyer, Bourne, and Hart (1992) tested the comparator account of overshadowing by following the conditioning phase with extinction of the overshadowing stimulus under conditions very similar to those used in the present experiments; they found no support for the comparator account. Consequently, we conclude that a "failure-ofacquisition" account - for example, the modified RescorlaWagner theory outlined above - provides a better explanation for the present results than any modified "failure-ofperformance" account.

Finally, it may be noted that the serial overshadowing effect studied here bears some similarity to another retroactive interference effect, known as retroactive cue interference (RCI), that has been reported in a number of studies using both human and rat participants (Escobar, Matute, \& Miller, 2001; Matute \& Pineño, 1998; Miguez, Cham, \& Miller, 2012). The procedure used to produce RCI is one in which subjects first learn to associate cue $\mathrm{X}$ with some outcome and, in a later session, learn to associate a second cue, $\mathrm{Y}$, with the same outcome. Acquisition of the second association can lead to a reduction in responding to $\mathrm{X}$ as compared to a control procedure in which, for example, $\mathrm{Y}$ is paired with a different outcome in the second stage. How RCI is best explained is still unclear, as is its relevance to the interference effects studied here given that RCI is attenuated if the common outcome is a biologically significant US (Escobar et al., 2001). Nonetheless, experimental comparisons between RCI and serial overshadowing may prove valuable in revealing common mechanisms. If the two effects were indeed mediated by a common mechanism, this would explain why the interference effect studied here is greater when the interfering stimulus is contiguous with the US, rather than contiguous with the target taste, because the former case produces a stronger association between the interfering taste and illness.

In conclusion, the present study confirms and extends previous evidence indicating that events, whether tastes or placement in a context, occurring shortly before a lithium injection produce greater overshadowing of the target aversion than events occurring earlier in the target-injection interval. It also highlights the difficulty met by current theories in explaining this consistent finding. 
Author notes The research reported here was supported by a grant from the Australian Research Council and the School of Psychology, University of Sydney.

\section{References}

Cannon, D. S., Best, M. R., Batson, J. D., Brown, E. R., Rubenstein, J. A., \& Carrell, L. E. (1985). Interfering with taste aversion learning in rats: The role of associative interference. Appetite, 6(1), 1-19.

Denniston, J. C., Savastano, H. C., \& Miller, R. R. (2001). The extended comparator hypothesis: Learning by contiguity. In R. R. Mowrer \& S. B. Klein (Eds.), Handbook of contemporary learning theory (pp. 65-117). Mahwah: Erlbaum.

Escobar, M., Matute, H., \& Miller, R. R. (2001). Cues trained apart compete for behavioral control in rats: Convergence with the associative interference literature. Journal of Experimental Psychology: General, 130, 97-115.

Kaye, H., Gambini, B., \& Mackintosh, N. J. (1988). A dissociation between one-trial overshadowing and the effect of a distractor on habituation. Quarterly Journal of Experimental Psychology, 40B, 3147.

Kwok, D. W. S., Livesey, E. J., \& Boakes, R. A. (2012). Serial overshadowing of taste aversion learning by stimuli preceding the target taste. Learning and Behaviour, 40(4), 427-438.

Lavin, M. J. (1976). The establishment of flavor-flavor associations using a sensory preconditioning training procedure. Learning and Motivation, 7, 173-183.

Leising, K. J., Garlick, D., \& Blaisdell, A. P. (2011). Overshadowing between landmarks on the touchscreen and in ARENA with pigeons. Journal of Experimental Psychology: Animal Behavior Processes, 37, 488-494.

Mackintosh, N. J. (1971). An analysis of overshadowing and blocking. Quarterly Journal of Experimental Psychology, 23, 118-125.
Mackintosh, N. J., \& Reese, B. (1979). One-trial overshadowing. Quarterly Journal of Experimental Psychology, 31, 519-526.

Matute, H., \& Pineño, O. (1998). Stimulus competition in the absence of compound conditioning. Animal Learning \& Behavior, 26, 3-14.

Miguez, G., Cham, H. X., \& Miller, R. R. (2012). Spontaneous recovery and $\mathrm{ABC}$ renewal from retroactive cue interference. Learning \& Behavior, 40, 42-53.

Pearce, J. M. (1987). A model for stimulus generalization in Pavlovian conditioning. Psychological Review, 94, 61-73.

Quinn, V. F., \& Colagiuri, B. (2015). Placebo interventions for nausea: A systematic review. Annals of Behavioral Medicine, 49(3), 449-462.

Rescorla, R. A., \& Wagner, A. R. (1972). A theory of Pavlovian conditioning: Variations in the effectiveness of reinforcement and nonreinforcement. In A. H. Black \& W. F. Prokasy (Eds.), Classical conditioning II: Current research and theories (pp. 64 99). New York: Appleton Century-Crofts.

Revusky, S. (1971). The role if interference in association over a delay. In W. K. Honig \& P. H. R. James (Eds.), Animal Memory (pp. 155213). New York: Academic Press.

Revusky, S., Parker, L. A., \& Coombes, S. (1977). Flavor aversion learning: Extinction of the aversion to an interfering flavor after conditioning does not affect the aversion to the reference flavor. Behavioral Biology, 19, 503-508.

Schachtman, T. R., Kasprow, W. J., Meyer, R. C., Bourne, M. J., \& Hart, J. A. (1992). Extinction of the overshadowing CS after overshadowing in conditioned taste aversion. Animal Learning \& Behavior, 20, 207-218.

Spetch, M. K. (1995). Overshadowing in landmark learning: Touchscreen studies with pigeons and humans. Journal of Experimental Psychology: Animal Behavior Processes, 21, 166-181.

Wagner, A. R. (1981). SOP: A model of automatic memory processing in animal behavior. In N. E. Spear \& R. R. Miller (Eds.), Informal processing in animals: Memory mechanisms (pp. 5-47). Hillsdale: Erlbaum Associates. 